\title{
Promover en los contextos de trabajo procesos de "Re-Skilling" y "Up-Skilling": el instrumento Ecvet
}

\author{
OREFICE, Carlo ${ }^{1}$; GURAZIU, Erina ${ }^{2}$
}

\section{RESUMEN}

De acuerdo con el Informe Future of Jobs Report (2016) del Foro Económico Mundial, estamos experimentando una transformación social, económica y productiva que involucra cada actividad humana y que está llevando las relaciones entre las profesiones y los roles profesionales a asumir nuevas trayectorias, así como a exigir a las organizaciones y a los propios trabajadores nuevas habilidades y formas de pensamiento "complejas". Para guiar este cambio, es esencial apoyar a las personas a través de procesos de mejora de calificaciones y capacitación para que puedan seguir siendo competitivos en el mercado laboral y garantizar condiciones de vida dignas.

A partir de estas premisas, este artículo tiene la intención de exponer una visión general del instrumento ECVET (European Credit system for Vocational Education and Training), un sistema europeo de créditos para la educación y la formación profesional que tiene como objetivo promover la empleabilidad de las personas de los países de la Unión Europea; por otro lado, se pregunta si las condiciones que hicieron posible adoptar este instrumento en Europa podrían transferirse a otros contextos laborales, sociales y económicos, o más bien a aquellos que pertenecen a los Países de la Alianza del Pacífico que actualmente participan en un específico proyecto transnacional Ilamado EMPLE-AP.

Palabras clave: resultados de aprendizaje, ECVET, empleabilidad, políticas de trabajo.

\section{Promoting "Re-skilling" and "Up-skilling" processes in working contexts: the Ecvet instrument}

\begin{abstract}
According to the Future of Jobs Report, World Economic Forum, (2016), we are experiencing a social, economic and productive transformation that involves every human activity and is leading the relationships between professions and professional roles to take on new paths, as well as demanding new skills and "complex" ways of thinking from organizations and workers themselves. To guide this change, it is essential to support people through processes of skills enhancement and training so that they can remain competitive in the labor market and ensure decent living conditions. Based on these premises, this article intends to give an overview of the ECVET instrument (European Credit System for Vocational Education and Training), a European credit system for vocational education and training that aims to promote the employability of people from European Union countries. On the other hand, it asks whether the conditions that made it possible to adopt this instrument in Europe could be transferred to other labor, social and economic contexts, or rather to those belonging to the Pacific Alliance Countries currently participating in a specific transnational project called EMPLE-AP.
\end{abstract}

Keywords: learning outcomes, ECVET, employability, labor policies

Universidad de Siena. ORCID:0000-0003-3502-3678. carlo.orefice@unisi.it

2Universidad de Florencia. ORCID: 0000-0003-3502-3678, erina.guraziu@unifi.it 


\section{INTRODUCCIÓN}

Según el Informe Future of Jobs Report (2016) del Foro Económico Mundial, estamos experimentando una transformación social, económica y productiva que involucra cada actividad humana y que está llevando las relaciones entre las profesiones y los roles profesionales a asumir nuevas trayectorias, así como a exigir a las organizaciones y a los propios trabajadores nuevas habilidades y formas de pensamiento "complejas". Para guiar este cambio - impulsado por los procesos de globalización y las TIC - es esencial apoyar a las personas a través de procesos de mejora de calificaciones (re-skilling) y capacitación (upskilling) para mantener su competitividad en el mercado laboral y garantizarse condiciones de vida dignas. En el campo educativo, la urgencia está en identificar modelos de capacitación apropiados que enfrenten estos desafíos y permitan a las personas actualizar sus habilidades.

En Europa, en 2009, el Parlamento Europeo y el Consejo establecieron un Sistema Europeo de Créditos para la Educación y Formación Profesional (ECVET) con el objetivo de fomentar una comunicación horizontal interconectada entre los países miembros con respecto a la transferencia y el reconocimiento mutuos de las habilidades profesionales adquiridas por individuos en diferentes contextos de aprendizaje (formal, informal y no formal), para facilitar la movilidad de las personas y por lo tanto, mejorar su empleabilidad.

A partir de estas consideraciones, está claro que uno de los desafíos emergentes en los sistemas universitarios, tanto europeos como de otros países, coincide con el apoyo a la activación y difusión dentro de la universidad de actividades de capacitación, capaces de apoyar la adquisición de aquellas competencias transversales consideradas estratégicas para aumentar la empleabilidad de los estudiantes (Cleary, Flynn y Thomasson, 2006). Definidas en taxonomías internacionales y nacionales con diferentes términos (habilidades blandas, competencias transversales, competencias clave, etc.), las competencias transversales se han convertido en uno de los temas centrales de la agenda de innovación educativa de las universidades, exhortando a los involucrados en el gobierno académico a responder tres preguntas clave: ¿cuáles son las habilidades transversales a desarrollar para promover la empleabilidad?; ¿a través de qué dispositivos de capacitación, la universidad puede apoyar la adquisición de estas habilidades?; ¿cómo describir las competencias para que puedan ser evaluadas y objetivamente reconocidas, tanto en el contexto académico como en el mundo laboral?

Como veremos, el sistema ECVET, parece significativo para responder estas preguntas, ya que favorece una planificación educativa que sigue un enfoque output-oriented (es decir, centrado en las competencias o los resultados del aprendizaje), en lugar de un enfoque tradicional input-oriented (basado en en contenido, duración, experiencia de aprendizaje, tipo de institución, etc.). Al adoptar esta perspectiva, la capacitación en habilidades es más capaz de proporcionar a los profesionales una capacidad "crítica" que es cada vez más necesaria para enfrentar los desafíos indicados, a través de la introducción de nuevos conceptos clave, como la concentración en el alumno con respecto a la ruta de capacitación y el enfoque en aprender en lugar de enseñar (Fabbri y Melacarne, 2015).

De hecho, si consideramos las transformaciones sociales, económicas y productivas a las que el Informe Future of Jobs Report (2016) se refiere como "reformuladoras" y "reorientadoras" de las relaciones entre profesiones y roles profesionales, es necesario acompañar a los profesionales a través de esta "desorientación" que parece ser de naturaleza económica y social, así como conectada con el nivel de significados y las habilidades que poseen. Es la propia Comisión Europea, con la Recomendación ECVET de 2009, la que refuerza esta necesidad cuando invita a los Estados miembros a adoptar un diseño educativo diseñado para la "unidad de resultados de aprendizaje", yendo así a perseguir "la creación de un sistema de crédito europeo para la educación y formación profesional (ECVET) destinado a facilitar la transferencia, el reconocimiento y la acumulación de resultados comprobados de aprendizaje para las personas interesadas en adquirir una calificación" (Recomendación 2009/C 155/02, p. 2).

Del mismo modo, el Consejo Europeo, en 2012, reitera que "la validación de los resultados del aprendizaje, es decir, el conocimiento, las habilidades y las competencias adquiridas a través del aprendizaje no formal e informal, puede desempeñar un papel importante en la mejora de la empleabilidad y la movilidad, así como en el aumento de motivación para el aprendizaje permanente, en particular en el caso de personas socialmente y económicamente 
desfavorecidas o menos calificadas" (Recomendación 2012/C 398/01, p. 1).

Siguiendo esos consejos, la adopción del concepto de "resultados de aprendizaje" aparece - en la medida en que se está discutiendo particularmente en forma significativa y está vinculada a un enfoque pedagógico "innovador" en la forma de concebir, describir y certificar el aprendizaje, y por lo tanto en repensar la misma educación y formación (Orefice, 2019). Es evidente, por lo tanto, que los resultados de aprendizaje de los que hablamos y que constituyen una calificación son independientes de los contextos de aprendizaje (que pueden ser formales, no formales o informales), y permiten promover, según lo tratado por la Recomendación ECVET, beneficios diversificados en términos de inclusión, creación de redes, innovación, transnacionalidad, enfoque bottomup de la educación.

A partir de este análisis, podemos afirmar que el sistema ECVET garantiza el continuo reskilling (reemplazar las habilidades), deskilling y upskilling (mejorar las habilidades) de las personas, en un continuo de mejora cualitativa de la oferta educativa que surge, también, como resultado de la iniciativa de las organizaciones en el mundo del trabajo para reconocer las habilidades transferidas a los individuos gracias al "aprendizaje experimental". Desde este punto de vista, este sistema contribuye a apoyar a las personas a adaptarse a un mercado laboral flexible y que cambia rápidamente, reduciendo la diferencia entre las personas que tienen una calificación formalmente reconocida y aquellas que solo pueden presumir de habilidades adquiridas en contextos no formal o informal.

Al tratar de responder a las preguntas antes formuladas, es importante, hacer una referencia al Proyecto EMPLE-AP ${ }^{3}$ - que se enfoca en la colaboración entre universidades e instituciones pertenecientes a varios países - parece particularmente útil. Este proyecto, ahora en una etapa avanzada de desarrollo, recuerda una serie de objetivos que están vinculados no solo a la necesidad de crear una oferta de capacitación relevante y al fortalecimiento de los programas académicos ofrecidos por las instituciones de educación superior de los países participantes, sino también, en un sentido más amplio, se refiere a una compleja red de desafíos que el futuro nos presenta. En esta dirección, los beneficios para las partes interesadas parecen ser múltiples y se convierten en un caso de prueba para los desafíos sociales y educativos mencionados anteriormente.

Las universidades que participan en el proyecto, de hecho, se benefician del uso de metodologías diversificadas (cuestionarios semiestructurados; entrevistas telefónicas; análisis de materiales presentes en los servicios de colocación; etcétera) para el análisis de colocación laboral, obteniendo así indicadores de empleabilidad que pueden ofrecer a sus estudiantes o empresas datos útiles sobre la empleabilidad de esa región/área específica.

En esta dirección, debe tenerse en cuenta que mejorar la empleabilidad de los graduados implica necesariamente conocer las necesidades de los sectores de actividad de los países de la Alianza, adaptar la educación a los mercados laborales existentes y llenar las brechas existentes en el capital humano, algo que está trayendo las diferentes regiones y actividades económicas a desarrollar un "mapa del tejido de las empresas de referencia" para analizar la situación del empleo. Finalmente, los estudiantes y graduados, o los principales actores del proyecto, se involucran a través de encuestas específicas y cuestionarios específicos, para que su participación sea activa, pero que los diferentes perfiles socio-académicos sean conocidos por desarrollar acciones sistémicas para su ventaja.

De una manera muy esquemática, dos parecen ser los retos más importantes a destacar en comparación con lo que se ha dicho hasta ahora. El primer desafío cuestiona la necesidad de "crear un sistema" entre las diversas universidades e instituciones involucradas en la promoción de la empleabilidad de los jóvenes, incluidas las instituciones de formación profesional y las empresas, garantizando así uno de los principales procesos de este nuevo siglo: el foco de las personas en la educación y la formación. De hecho, la necesidad de combinar criterios, como el de presentar problemas y presentar soluciones compartidas con respecto al desempleo de los jóvenes, nos recuerda que la cuestión del empleo es tarea y responsabilidad de todos y allana el camino hacia una acción concertada en apoyo de las políticas públicas.

La Universidad de Siena ha estado involucrada desde el 2017 en el Proyecto llamado "EMPLE-AP - Observatorio para la inserción laboral y fortalecimiento de la empleabilidad en países de la Alianza del Pacífico" (http://www.emple-ap.com/es). Cofinanciado por el programa Erasmus +, el Proyecto tiene como objetivo contribuir a mejorar los índices de empleabilidad y la colocación laboral de graduados de instituciones de educación superior en los países de la Alianza del Pacífico (México, Colombia, Perú y Chile), con el fin de transferir el conocimiento y las buenas prácticas entre las universidades y centros de estos países y las universidades de la Unión Europea que han logrado un progreso significativo en los sectores indicados (Francia, Italia, Portugal, España). 
El esfuerzo consiste en enfrentar algunas debilidades estructurales de baja productividad y la debilidad del desarrollo y la diversificación de producción que los países, (no sólo los de la Alianza del Pacífico), están experimentando en este momento, así como a invertir más en educación y formación profesional.

Desde este punto de vista, el contexto cada vez más "líquido" (Bauman, 2011) del mercado laboral, caracterizado por una dimensión global e interconexiones cada vez más diversificadas y complejas, ha dado un golpe a las teorías y prácticas educativas en las que, hasta hace unas décadas, se habían construido los principales modelos fundamentales de la pedagogía del trabajo en Europa, dirigiendo la acción hacia políticas laborales que se centran en la persona, consideradas en su singularidad y con necesidades no atribuibles a esquemas predeterminados por parte de la economía y del mercado.

El segundo desafío recuerda el tema de la "precariedad juvenil", que en los países de la Alianza del Pacífico, así como en Europa (aunque en diferentes coordenadas culturales y políticas), a veces se traduce en un comportamiento problemático y antisocial. Si bien los comportamientos desviados y problemáticos tienen naturalmente diferentes causas (ausencia de valores, pobreza, abandono, inquietud social, falta de perspectivas, etc.), a través del estudio comparativo de las situaciones laborales de los jóvenes que los diferentes socios del Proyecto EMPLE-AP están llevando a cabo en sus investigaciones, es posible argumentar que un bajo nivel de educación, una falta de educación con respecto a la orientación laboral y el círculo vicioso de la pobreza que obliga a una búsqueda cada vez más temprana de trabajo abandonando los estudios ( $y$ por lo tanto a permanecer en la pobreza), están directamente vinculados a una mayor vulnerabilidad social y a los efectos psicosociales a los que se refiere (OIT, 2017).

En última instancia, al tomar nota de la profunda revisión de los modelos de bienestar que se han desarrollado y consolidado en todos los países involucrados en el Proyecto (europeos y no europeos), EMPLE-AP se refiere en gran medida al tema de qué competencias son las más adecuadas para responder a los múltiples desafíos emergentes indicados, cuestionando y problematizando el tema del trabajo, de la producción, de la solidaridad y protección social de los sujetos más vulnerables (Orefice y Melacarne, 2018).

\section{CONSIDERACIONES FINALES}

La necesidad de aclarar mejor los principios y conceptos definidos en el contexto de las políticas europeas, con respecto al reconocimiento, evaluación y certificación de los resultados de aprendizaje adquiridos por los adultos durante su vida profesional, es posible destacar algunos resultados y beneficios logrados con la adopción del sistema ECVET en algunos países europeos, así como algunos problemas.

Como ventajas, a nivel personal de los individuos, los principales beneficios parecen ser la posibilidad de acortar los caminos para el reconocimiento de las calificaciones formales mantenidas; la posibilidad de reconstruir los diversos momentos formativos y profesionales realizados, mejorar la herencia del aprendizaje adquirido y diseñar un camino dotado de significado que contribuya a fortalecer la identidad profesional; volver a motivarse y reorientarse, mejorar la autoestima y la autoconfianza y estimular una nueva planificación profesional y/o de capacitación. Para los sistemas educativos, podemos destacar la posibilidad de construir un lenguaje común para los mundos de la educación, la capacitación y el trabajo, fomentando el diálogo y la colaboración; personalizar las intervenciones de capacitación; favorecer el regreso a la capacitación de aquellos que no han completado las rutas de estudio, a través del reconocimiento y mejora, en el plan de formación, de la experiencia realizada.

También hay limitaciones y aspectos problemáticos a los que se refiere el sistema ECVE, entre estos, podemos destacar la comparabilidad de los diferentes tipos de aprendizaje (experiencial y académico); la validez y fiabilidad de los sistemas de evaluación; la profesionalidad de quienes trabajan en el servicio; los recursos necesarios; el papel de la universidad. En particular, muchas críticas se han dirigido a las formas en que se construyeron las pruebas de acreditación, a menudo reduciéndolas a una mera verificación de las competencias de las personas, con poca atención al aspecto reflexivo y constructivo de la cartera de habilidades y al acompañamiento de las asignaturas en el mundo laboral. A pesar de su diferenciación y limitaciones, los resultados 
logrados con la adopción del sistema ECVET en algunos países europeos, así como aquellos que el proyecto EMPLE-AP ha llevado a cabo en los últimos años, permiten obtener las primeras conclusiones provisionales, entre las que están:

La implementación de las competencias y habilidades de los estudiantes en transición de la escuela a la universidad y de la universidad al mundo laboral, cuestiona las epistemologías y las teorías interpretativas que deben conectar las disciplinas y sus conocimientos, así como crear otros nuevos. El desafío es ser capaz de leer y gestionar las conexiones entre realidades aparentemente extrañas, pero también ser capaz de poner en evidencia conocimientos que durante mucho tiempo se han mantenido alejados o desconocidos, por lo tanto, superar la naturaleza científica del conocimiento de disciplinas separadas y teorías definitivas es una suposición necesaria, para explorar una realidad que se manifiesta cada vez más compleja, así como para promover habilidades que son realmente útiles para la empleabilidad.

Parece cada vez más fuerte la necesidad que el sistema de educación superior integre su propuesta de capacitación disciplinaria con aquellos métodos de enseñanza activa que permiten el desarrollo de habilidades blandas (o "habilidades transversales") como un bagaje personal de habilidades y características, lo que permiten al individuo moverse, en el contexto de pertenencia, de la mejor manera posible. Desde este punto de vista, es necesario realizar cada vez más una didáctica que intercepte los problemas que enfrentarán los jóvenes al final de sus estudios para que sea capaz de introducir, durante el curso universitario, conocimientos, experiencias y buenas prácticas que le ayude a prefigurar la futura profesión; de manera similar, parece necesario promover iniciativas de capacitación (cursos, maestrías) para maestros de secundaria.

Esto pone en tela de juicio a las universidades involucradas en los procesos de "recalificación" y "educación superior", que deberían contribuir cada vez más a la compleja investigación científica en términos de modelos abiertos, que se refieren a niveles y campos de la realidad particular desde el punto de vista de la transdisciplinariedad.

Es posible argumentar que el sistema ECVET al tratar con el reconocimiento, la evaluación y la certificación de los resultados de aprendizaje adquiridos por los adultos durante su trayectoria profesional, puede constituir una "buena práctica" que puede replicarse en el contexto de la Alianza del Pacifico para promover la empleabilidad, ya que parece capaz de favorecer:

- la inclusión social: al centrarse en los resultados de aprendizaje que son realmente demostrables y validados al final de la ruta de aprendizaje, el individuo se coloca en el centro del proceso educativo y su trayectoria de vida se reconoce como un flujo continuo de adquisición de habilidades; es decir, compuesto de contextos de aprendizaje formales, no formales e informales, con resultados demostrables, observables y documentables, así como reconocibles entre países.

- las redes entre el mundo del trabajo, los interlocutores sociales y el sector de la educación y la formación: con el fin de reconocer las habilidades adquiridas por los individuos a lo largo de sus vidas (tanto en contextos no formales como informales), ECVET se convierte en una herramienta de diálogo que facilita la asociación y la confianza mutua entre las instituciones responsables de las calificaciones y las organizaciones en el tejido económico a nivel transnacional. Además, este sistema tiene como objetivo mejorar la compatibilidad, comparabilidad y complementariedad entre los sistemas de crédito utilizados en la educación y formación profesional y el sistema europeo de acumulación y transferencia de créditos (ECTS), contribuyendo así a una mayor permeabilidad entre sistemas y entre países.

o la innovación: el sistema ECVET se traduce en un cambio sustancial en el paradigma de aprendizaje/enseñanza. Pasamos de una visión tradicional, basada en los contenidos, a un enfoque basado en los resultados, acumulados por el individuo durante el camino de su vida en diferentes contextos. Por lo tanto, el individuo se coloca en el centro de la ruta de entrenamiento $y$, gracias al sistema ECVET, puede ser evaluado y así reconocer las habilidades adquiridas a través de la experiencia. El enfoque basado en los resultados también permite un mayor enfoque en la calidad de la capacitación: el contenido no se evalúa, sino la satisfacción de los resultados esperados en términos del conocimiento, las habilidades y las competencias que la persona demuestra poseer.

- la transnacionalidad: al adoptar el sistema, se prevé la creación de un espacio de aprendizaje permanente sin fronteras, donde se garantice la transparencia de los resultados, la movilidad transnacional de los ciudadanos, la portabilidad 
de las calificaciones entre los Estados miembros y el reconocimiento de las unidades de los resultados de aprendizaje adquiridos en otros países en contextos formales, no formales e informales. Este parece ser el único intento, a nivel mundial, de armonización y diálogo entre los marcos nacionales de calificaciones para reconocer y certificar las habilidades profesionales de los individuos, adquiridas también en contextos profesionales y no profesionales, de acuerdo con una perspectiva transnacional. Como veremos, a partir de aquí surge la necesidad y el desafío de transferir el modelo del contexto europeo a un contexto transcontinental, a fin de apoyar la movilidad de las personas y su dignidad humana y profesional.

o un enfoque bottom-up: a través de principios transparentes de aseguramiento de la calidad, el intercambio de información y el desarrollo de asociaciones entre instituciones y sujetos competentes, contribuimos a la creación de un clima de confianza mutua.

\section{REFERENCIAS BIBLIOGRÁFICAS}

Andersson, P. F. (s.d.). Introducing research on recognition of prior learning. International Journal of Lifelong Education, 32, p. 405-411

Bauman Z. (2011) Modernità liquida. Roma-Bari: Laterza

Cleary M., Flynn R., Thomasson S. (2006) Employability Skills: From Framework to Practice-An Introductory Guide for Trainers and Assessors(http://www.fmpllen.com.au/uploads/ 1/2/9/9/12992035/employability_skills_from_fr amework_to_practice_an_introductory_guide_ for_trainers_and_assessors.pdf)

Consejo Europeo de Lisboa. (2000, Marzo 23 y 24). European Parliament. Tratto da Conclusiones de la $\begin{array}{lllllllllll} & \mathrm{r} & \mathrm{s} & \mathrm{i} & \mathrm{d} & \mathrm{e} & \mathrm{n} & \mathrm{c} & \mathrm{i} & \mathrm{a} & \text { : }\end{array}$ http://www.europarl.europa.eu/summits/lis1_es.htm

Fabbri L., Orefice C., Bianchi F. (2018) Favorire I'innovazione della governance e delle politiche universitarie. Riflessioni a margine della proposta PRIN/GEO e di un prossimo convegno. In: M. Michelini (a cura di). Riflessioni sull'innovazione didattica universitaria: interventi alla Tavola rotonda GEO (30 giugno 2017). Udine: Editrice Universitaria Udinese, pp. $128-130$

Fabbri L., Melacarne C. (2015) Apprendere a Scuola. Metodologie attive di sviluppo e dispositivi riflessivi. Milano: FrancoAngeli

Hargreaves, J. e. (2006). Recognition of Prior Learning: At a Glance. Adelaide: NCVER

Melacarne C., Orefice C., Giampaolo M. (2018) Supporting key competences and soft skills in Higher Education. In: P. Federighi. Educazione in età adulta: ricerche, politiche, luoghi e professioni. Firenze: FUP, pp. 181-186

OIT - Organización Internacional del Trabajo (2017) Panorama Laboral 2017 - América Latina y el Caribe
Orefice C. (2019). SDG 4: Quality Education, in J-E. Baños, C. Orefice, F. Bianchi, S. Costantini: Good Health, Quality Education, Sustainable Communities, Human Rights. The scientific contribution of Italian UNESCO Chairs and partners to SDGs 2030, FUP, pp. 109-112

Orefice C., Guraziu E.(2018)Making educational planning: skills, methodologies, experiences. FORMARE - Open Journal per la formazione in rete, $3 / 18$, pp. 142-152

Orefice C., Melacarne C.(2018) Soft skills e occupabilità giovanile. In: S. Ulivieri (a cura di). Le emergenze educative della società contemporanea. Progetti e proposte per il cambiamento. Lecce: Pensa MultiMedia, pp. 491-497

Perulli, E. (2010). Trasparenza e certificazione di qualifiche e competenze: esperienze nazionali e prospettive europee. Quaderni di Economia del Lavoro(93), p. 13-26Raccomandazione 2009/C 155/02 del Parlamento Europeo e del Consiglio, 18 giugno 2009 sull'istituzione di un sistema europeo di crediti per l'istruzione e la formazione professionale (ECVET)

Raccomandazione 2012/C 398/01 del Consiglio Europeo, 20 dicembre 2012 sulla convalida dell'apprendimento non formale e informale

Recomendación 2017/C 189/03. (s.d.). Consejo de la Unión Europea. Tratto da eur-lex: https: / / eur-Iex.europa.eu / legalcontent/ES/TXT/HTML/?uri=CELEX:32017H061 $5(01)$ \&from $=\mathrm{EN}$

Romano A., Orefice C.(2018) Come coprogettare spazi per l'inclusione nei contesti dell'Higher Education. Uno studio di caso. ITALIAN JOURNAL OF SPECIAL EDUCATION FOR INCLUSION, anno VI, vol. 2, pp. 205-215

Villaba-Garcia, E., Chakroun, B., (2019) RVA that counts: what data do we need to nurture 
recognition, validation and accreditation of prior learning? In: Cedefop, ETF, UNESCO, UIL (a cura di), Global Inventory of regional and national qualitifacion frameworks 2019) pg. 45 - 59
World Economic Forum (2016) The future of jobs report (http://reports.weforum.org/future-ofjobs-2016/)

Recibido: 05 marzo 2019 | Aceptado: 10 junio 2019 